\title{
Thrombolysis, the general practitioner, and the electrocardiogram
}

The British Heart Foundation Working Group on the early management of myocardial infarction has stated that-"General practitioners initiating thrombolytic treatment outside hospital need to be fully aware of the indications, contraindications, and side effects of such treatment and should have a defibrillator available. They should confirm the diagnosis by a 12-lead electrocardiogram."1

\section{Should general practitioners give thrombolysis?}

General practitioners participating in the GREAT study responded quickly to patients with suspected myocardial infarction and administered thrombolytic drugs reliably, safely, and effectively some two hours before they would have received this treatment in hospital. ${ }^{2}$ The British Heart Foundation Working Group concluded that it is appropriate for general practitioners to administer these drugs if, by doing so, patients with definite myocardial infarction will receive them an hour or more earlier than they otherwise would. ${ }^{1}$ In many areas, this proviso will not apply because patients will be treated more promptly if they send for an ambulance rather than for their doctor.

Any general practitioner who plans to give such treatment should know its indications and contraindications, be able to record and interpret an electrocardiogram, and have access to a defibrillator. But what if these conditions do not obtain, especially if a general practitioner cannot for one reason or another obtain or read an electrocardiogram?

\section{Is the electrocardiogram a useful guide to thrombolytic therapy?}

The electrocardiogram is an invaluable tool for determining the probable effectiveness of thrombolytic therapy. If the clinical picture is one of myocardial infarction within 6 hours of its onset and the electrocardiogram shows ST elevation or bundle branch block, thrombolysis will save the life of some $3 \%$ of patients to whom it is given. ${ }^{3}$ If the electrocardiogram is normal or shows various less specific abnormalities (including ST depression) the mortality benefit, if any, is probably less than $1 \%$.

\section{But an electrocardiogram taken soon after the} onset of infarction is not reliable

The electrocardiogram may be normal or non-diagnostic soon after the onset of infarction, yet show typical changes later. ${ }^{4}$ This emphasises the need to repeat the electrocardiogram frequently in those with a high likelihood of infarction, rather than to use thrombolytic therapy when the diagnosis is so uncertain.

ST elevation can, of course, be misleading. It may be the residue of old infarction or a transient feature of ischaemia in the absence of infarction. When the ST elevation disappears after the administration of a thrombolytic our more enthusiastic colleagues are apt to claim that they have aborted an infarct; they may have aborted a phantom infarct. None the less, it is as well to retain
ST elevation as an important indication for thrombolysis because the benefits of giving it in these circumstances greatly outweigh the risks.

\section{Has no electrocardiogram the same significance as} a normal electrocardiogram?

It has often been assumed that, because it is inappropriate to give thrombolysis if the electrocardiogram is normal or near normal, it is wrong to give such treatment if there is no electrocardiogram. This does not follow, because the probability of infarction benefiting from thrombolysis is different in the two circumstances. If a patient with known ischaemic heart disease has experienced unstable angina leading to an episode of severe typical infarction pain, there is perhaps a $50 \%$ chance that the electrocardiogram would show ST elevation or bundle branch block.

\section{How accurate is the diagnosis of myocardial} infarction in the absence of an electrocardiogram? In a study of general practitioners in the Taunton area, the diagnosis of myocardial infarction was confirmed in 36 of the 100 patients in whom it was suspected. ${ }^{5}$ The practitioners rated their certainty of the diagnosis on a 1-10 scale. Only $46 \%$ of those graded 10 (certain of the diagnosis) proved to have a myocardial infarction. In the GREAT study, in which an electrocardiogram was not a requirement for entry into the trial, $61 \%$ of those recruited were finally classified as having definite or probable infarcts. However, because the general practitioners recorded an electrocardiogram at the time of entry, it is difficult to know how they were influenced by its findings.

Perhaps a more relevant question here is not how accurate general practitioners without electrocardiograms are in diagnosing all infarctions, but how accurate they are in recognising those infarctions for whom thrombolysis should be given. In the study of Gemmill et al from Glasgow only 18 out of 49 patients whom the practitioner thought appropriate for thrombolysis on clinical grounds were found in hospital to have the indications for it. ${ }^{6}$ The choice of patients for thrombolysis was not improved by trying to record an electrocardiogram; these practitioners had not, however, received instruction on interpreting electrocardiograms. More evidence is needed on the accuracy of suitably trained general practitioners in recognising suitable cases with and without an electrocardiogram before one could recommend that they should administer thrombolysis on clinical suspicion alone.

\section{What are the risks of inappropriate thrombolysis?}

Thrombolysis can cause serious adverse effects. Some of these, such as reperfusion ventricular fibrillation and early rupture, are peculiar to patients with recent infarction, but the more common and serious bleeding complications, especially haemorrhagic stroke, are as common in patients without infarction as they are in patients with 
it. This risk is in the region of $1 \%$ and would nullify any benefit that would be seen in infarction patients without ST elevation or bundle branch block. It is highly undesirable to expose patients without infarction to such a risk, particularly those who might have disorders such as dissection of the aorta or pericarditis, in whom bleeding is particularly hazardous.

\section{Can and should general practitioners read electrocardiograms?}

There is a widespread belief, at least among cardiologists, that general practitioners cannot read electrocardiograms, and evidence has been provided in support of this view. A sample of practitioners in the Merseyside area was found to be incapable of accurately interpreting records of myocardial infarction, ${ }^{7}$ although there and elsewhere general practitioners have been shown to be good at recognising a normal electrocardiogram. ${ }^{8}$ Waine, Hannaford, and Kay pointed out that few of the practitioners in the Merseyside study had any intention of giving thrombolytic therapy and were, therefore, not highly motivated to know about the criteria for its administration. ${ }^{9}$ Furthermore, it is certainly not important that practitioners should identify the precise site of the infarction, and it was not surprising they had difficulty in recognising anterior infarction in the presence of right bundle branch block-a diagnosis that often defeats MRCP candidates. Another important issue was identified by Gemmill et al in Glasgow. ${ }^{6}$ In this study, general practitioners obtained satisfactory electrocardiograms in only $60 \%$ of cases. This may have been owing to incorrect use of the machine; electrical interference seems to have been a major problem, as were patient movement and tremor artefact. These difficulties can be overcome in most cases and do not seem to have troubled the GREAT investigators.

Of course, there are exceptional general practitioners who have trained themselves to be competent in electrocardiography; but they are a small minority. But is it so difficult? Older colleagues will remember that until the mid-sixties, it was unthinkable for nurses to read electrocardiograms; indeed, one had to undertake years of postgraduate training before one could be entrusted to report on electrocardiograms for fellow physicians. Subsequently, nurses became extremely adept at electrocardiographic diagnosis; now coronary care nurses are much better than many doctors in reading electrocardiograms. Their competence depends partly upon their motivation and partly upon experience with pattern recognition. The real problem that faces general practitioners is not so much the training but the fact that they see acute myocardial infarction so infrequently-on average 1-4 times a year. Even general practitioners with electrocardiographs do not always use them in emergencies.

What do general practitioners need to know about the electrocardiogram in the context of suspected infarction?
In order to use thrombolytic treatment they must be able to recognise ST elevation and bundle branch block reliably. Teaching should concentrate on this, as well as on the recognition of ventricular fibrillation. This does not require expensive lectures and demonstrations; self-learning methods are perfectly satisfactory. In group practices, there is usually one partner who is or can become competent in electrocardiographic interpretation and act as mentor for the other members of the practice.

An alternative approach is to use computer diagnosis or trans-telephonic communication, but these are not realistic options for general practice in the United Kingdom in the immediate future.

\section{Conclusion}

General practitioners should be encouraged to use a thrombolytic if, by doing so, patients with almost certain infarction will receive treatment an hour or more earlier than they would otherwise. They should always attempt to record an electrocardiogram. If they are successful, they should give thrombolytics only to patients with ST elevation or bundle branch block. If, for some reason, they are unable to obtain or interpret the electrocardiogram, they must weigh up the likelihood of the patient having infarction in the light of the history and physical signs. Thus, if a patient with known ischaemic heart disease develops typical severe chest pain that is unresponsive to nitrates and requires an opioid, and if there are clinical features, such as hypotension or dyspnoea, that suggest infarction rather than angina, there may be a case for giving a thrombolytic. Such features are infrequent in patients presenting outside hospital with suspected infarction; in their absence thrombolysis should be given only to those with the recognised electrocardiographic characteristics.

Flat 1, 7 Netherhall Gardens,

DESMOND G JULIAN London NW3 $5 R N$

1 Weston CFM, Penny WJ, Julian DG on behalf of the British Heart Foundation Working Group. Guidelines for the early management of patients with myocardial infarction. $B M \mathcal{F} 1994 ; 308: 767-71$

2 GREAT Group. Feasibility, safety, and efficacy of domiciliary thrombolysis by general practitioners: Grampian region early anistreplase trail. BMF 1992;305:548-53.

3 Fibrinolytic Therapy Trialists (FTT) Collaborative Group. Indications for fibrinolytic therapy in suspected acute myocardial infarction: collaborative overview and major morbidity results for all randomised trials of more than 1000 patients. Lancet 1994;343:311-22.

4 Adams J, Trent R, Rawles J on behalf of the GREAT Group. Earliest electrocardiographic evidence of myocardial infarction: implications for thrombolytic treatment. $B M \mathcal{F}$ 1993;307:409-13.

5 Rule S, Brooksby P, Sanderson J. Use of thrombolysis for acute myocardial infarction by general practitioners. Postgrad Med f 1993;69:190-3.

6 Gemmill JD, Lifson WK, Rae AP, Hillis WS, Dunn FG. Assessment by general practitioners of suitability of thrombolysis in patients with suspected acute myocardial infarction. Br Heart $\mathcal{f}$ 1993;70:503-6.

7 McCrea WA, Saltissi S. Electrocardiogram interpretation in general practice: relevance to prehospital thrombolysis. Br Heart $\mathcal{F}$ 1993;70:219-25.

8 Colquhoun MC. General practitioners and the treatment of myocardial infarction: the place of thrombolytic treatment. $B r$ Heart $\mathcal{f} 1993 ; 70$ : 215-7.

9 Waine C, Hannaford P, Kay C. Early thrombolysis therapy: some issues facing general practitioners. Br Heart $\mathcal{f}$ 1993;70:218. 